\title{
MECHANISMS OF PERCEPTUAL LEARNING
}

\author{
ZHONG-LIN LU ${ }^{1} *$ and BARBARA ANNE DOSHER ${ }^{2}$ \\ ${ }^{1}$ Department of Psychology, University of Southern California \\ Los Angeles, CA, USA \\ ${ }^{2}$ Department of Cognitive Sciences, University of California \\ Irvine, CA, USA
}

What is learned in perceptual learning? How does perceptual learning change the perceptual system? We investigate these questions using a systems analysis of the perceptual system during the course of perceptual learning using psychophysical methods and models of the observer. Effects of perceptual learning on an observer's performance are characterized by external noise tests within the framework of noisy observer models. We find evidence that two independent mechanisms, external noise exclusion and stimulus enhancement support perceptual learning across a range of tasks. We suggest that both mechanisms may reflect re-weighting of stable early sensory representations.

Keywords: perceptual template model; stimulus enhancement; external noise exclusion; multiplicative noise reduction

\section{INTRODUCTION}

Perceptual learning in adult human observers has been documented in a wide range of perceptual tasks (Ahissar and Hochstein, 1996; Ball and Sekuler, 1982; Beard, Levi and Reich, 1995; DeValois, 1977; Dosher and Lu, 1998, 1999; Fahle and Edelman, 1993; Fine and Jacobs, 2000; Fiorentini and Berardi, 1980, 1981; Furmanski and Engel, 2000; Karni and Sagi, 1991, 1993; Mayer, 1983; McKee and Westheimer, 1978; Mollon and Danilova, 1996;

* Corresponding author; Department of Psychology, SGM 501, University of Southern California, Los Angeles, CA 90089-1061. Phone: (213) 740-2282; fax: (213) 746-9082; E-mail: zhonglin@usc.edu 
Ramachandran and Braddick, 1973; Sagi and Tanne, 1994; Shiu and Pashler, 1992; Vogels and Orban, 1985). Most studies of perceptual learning have investigated transfer or lack of transfer of perceptual learning to modified forms of the same task or to different, related tasks (Ahissar and Hochstein, 1996, 1997; Ahissar et al., 1998; Ball and Sekuler, 1987; Berardi and Fiorentini, 1987; Dorais and Sagi, 1997; Fiorentini and Berardi, 1980, 1981; Karni and Sagi, 1993; Liu and Vaina, 1998; Poggio, Fahle and Edelman, 1992; Ramachandran and Braddick, 1973; Rubenstein and Sagi, 1993; Saarinen and Levi, 1995; Schoups, Vogels and Orban, 1995; Shiu and Pashler, 1992). In such studies, the generalizability of the learning is usually assessed in the end of training, the character and the locus of learning are inferred from the extent of transfer, but task-relevant changes to the perceptual system during learning itself are rarely assessed directly.

What is learned during perceptual learning? Or, what aspect of information processing is improved through perceptual learning? These questions have been increasingly investigated in psychophysics (Chung, Levi and Tjan, 2005; Dosher and Lu, 1998, 1999; Gold, Bennett and Sekuler, 1999; Saarinen and Levi, 1995), neurophysiology (Crist, Li and Gilbert, 2001; Ghose, Yang and Maunsell, 2002; Schoups et al., 2001), brain imaging (Schiltz et al., 1999; Schwartz, Maquet and Frith, 2002), and patient studies (Fahle and Daum, 2002; Lu et al., 2008). In this review, we focus on the theoretical framework and psychophysical studies that address these questions by analyzing how perceptual inefficiencies improve over the course of perceptual learning. Perceptual inefficiencies are attributed to three limitations in perceptual processes: an imperfect perceptual template, internal additive noise, and multiplicative noise. Systematic measurements of human performance as a function of both the amount of external noise added to the signal stimulus and the length of training received by the observers enable us to distinguish three mechanisms of perceptual learning: external noise exclusion, stimulus enhancement, and internal multiplicative noise reduction.

\section{THE THEORETICAL FRAMEWORK}

In signal processing, there are three ways to improve the signal to noise ratio: amplification, improved filtering, and modified gain control. Similar principles of contrast gain, re-tuning of cellular signal selectivity, and reduced contrast-gain have also been demonstrated in single unit neurophysiology (Moran and Desimone, 1985; Reynolds, Pasternak and Desimone, 2000; Treue and Maunsell, 1999). Motivated by the principles in signal processing and neurophysiology, we developed the external noise plus attention paradigm and a theoretical framework based on the Perceptual Template Model (PTM) to distinguish perceptual mechanisms of attention and perceptual learning (Lu and Dosher, 1998; Dosher and Lu, 1998; see $\mathrm{Lu}$ and Dosher, 2008 for a recent review). 


\subsection{The external noise paradigm}

Perceptual performance is limited by such factors as intrinsic stimulus variability, receptor sampling errors, randomness of neural responses, and loss of information during neural transmission. At an overall system level, these inefficiencies can be quantified in terms of a noisy observer limited by equivalent internal noise - random internal noise necessary to produce the degree of inefficiency exhibited by the perceptual system (e.g. Dosher and Lu, 1999; Pelli, 1981). The amount of equivalent internal noise is estimated by systematically manipulating the amount of external noise (like TV snow or auditory white noise) added to the signal stimulus and observing how threshold - signal stimulus energy required for an observer to maintain a given performance level - depends on the amount of external noise (see $\mathrm{Lu}$ and Dosher, 2008 for a review). These methods characterize the overall limitations of the perceptual system, and allow comparisons of the efficiency of the perceptual system in different perceptual tasks. In fact, specification of internal noise has become a requirement of any computational model of human perception (Sperling, 1989).

\subsection{The perceptual template model}

The perceptual template model (Fig. la) consists of five components: (1) A perceptual template with certain tuning characteristics (e.g. a spatial frequency filter $F(f)$ with a center frequency and a bandwidth such that a range of frequencies adjacent to the center frequency pass

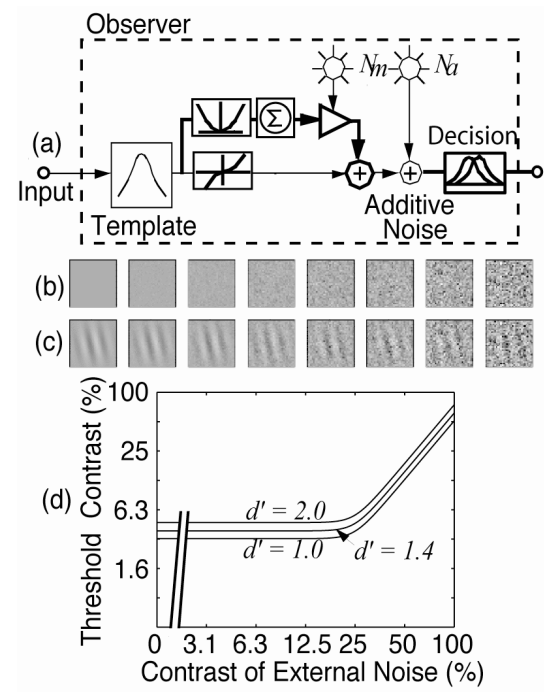

Figure 1. (a) A noisy perceptual template model. (b) Samples of eight levels of external noise. (c) A Gabor signal embedded in the external noises shown in (b). (c) Simulated threshold versus external noise contrast $(\mathrm{TvC})$ functions for a perceptual template model at three criterion performance levels

$$
\left(d^{\prime}=1.0,1.4,2.0\right) \text {. }
$$


through with smaller gains). The template is normalized such that it passes the noise with gain 1.0 and the signal stimulus with gain $\beta$. (2) A nonlinear transducer function with the form: output = sign(input) $\|$ input $\|^{\gamma}$. (3) A multiplicative internal noise that is Gaussian distributed with mean 0 and a standard deviation that is proportional (with a coefficient of $N_{m}$ ) to the total energy in the input stimulus. Multiplicative noise is a natural way of characterizing tasks in which, for example, perceived sensory variability, or perceived differences, are proportional to signal strength (Weber-law situations). (4) An independent additive internal noise that is Gaussian distributed with mean 0 and a fixed standard deviation $N_{a}$. The existence of an absolute sensory threshold for every perceptual process suggests that the perceptual system is limited by an additive noise whose amplitude is independent of the amount of input. (5) A decision process that operates on the noisy internal representation of the stimulus. Depending on the task, the decision could reflect either detection or discrimination, and could take the form of either N-alternative forced choice or "yes"/"no", possibly with confidence ratings, and uses a standard signal detection model for each task.

The perceptual limitations of the observer can be characterized by systematically manipulating the amount of external noise added to the signal stimulus (Figs. 1b, c) and observing how threshold - signal stimulus energy required for an observer to maintain a given performance level - depends on the amount of external noise (the threshold versus contrast, or TvC, function; Fig. 1d). In a typical application, the model parameters, $N_{a}, N_{m}, \beta$, and $\gamma$, are unknown quantities that can be estimated from $\mathrm{TvC}$ data such as those in Figure $1 d$ by nonlinear estimation techniques, or, alternatively, simple equations can be derived which allow us to compute estimates of several of the parameters from certain relations in the data. Two or three measured threshold levels are required (see Dosher and Lu, 1999; Lu and Dosher, 1999, for details). Although this characterization does not distinguish between various sources of the inefficiency, it does allow us to quantify the overall efficiency of the perceptual system, and to compare the efficiency of the perceptual system in different perceptual tasks.

\subsection{The external noise plus perceptual learning paradigm and signatures of perceptual learning mechanisms}

The theoretical performance signatures of mechanisms of perceptual learning (Dosher and Lu, 1998, 1999) can be derived by studying the possible ways training or practice can affect various components of the PTM and generate model $\mathrm{TvC}$ functions for proposed mechanisms of perceptual learning: stimulus enhancement, external noise exclusion, and internal multiplicative noise reduction (Fig. 2).

\subsubsection{Stimulus enhancement}

Enhanced performance due to perceptual learning in clear (noise-free) conditions corresponds to claims of perceptual enhancement. In the context of the PTM, stimulus enhancement is mathematically equivalent to internal additive noise reduction, or equivalently, to relative amplification of the stimulus. The behavioral signature for this mechanism is perfor- 
mance improvement (reduced thresholds or lower curves) in the region of low or zero external noise (Fig. 2a). Stimulus enhancement has no benefits for performance in high external noise because external noise is the primary limiting factor on performance, and enhancement affects both the signal and the external noise in the input stimulus in the same way.
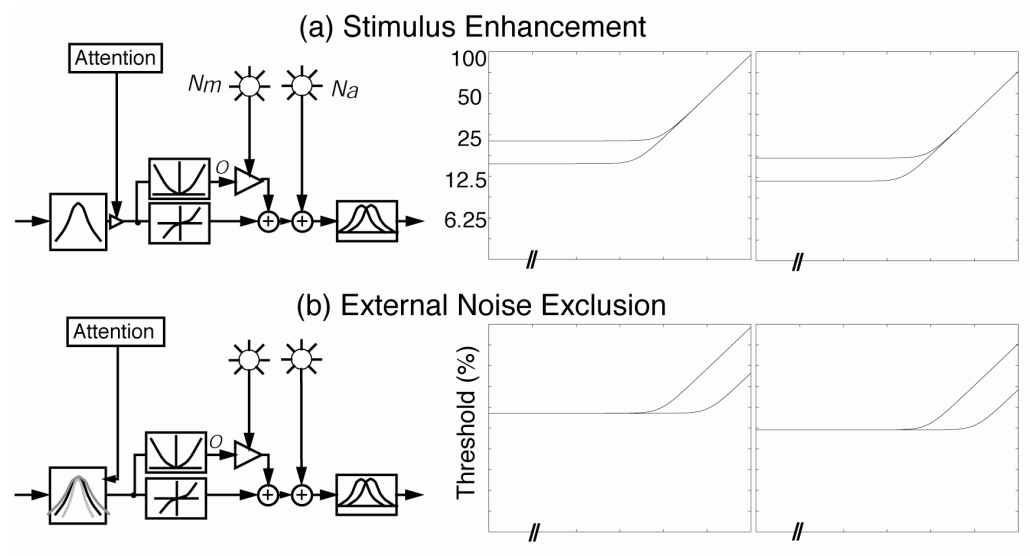

(c) Multiplicative Noise Reduction

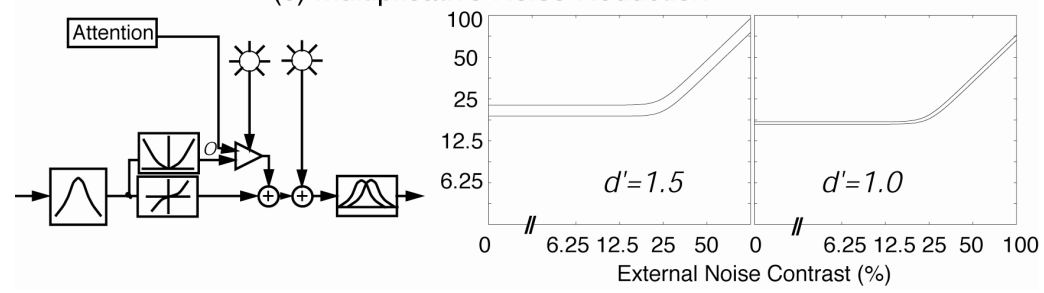

Figure 2. Signature performance patterns for three mechanisms of attention within the framework of a perceptual template model (PTM) at two performance criterion levels ( $d^{\prime}=1.5$ and 1.0): (a) Stimulus enhancement. It only improves performance in zero or low external noise. (b) External noise exclusion. It only modulates performance at high levels of external noise. (c) Internal multiplicative noise reduction. It affects performance at all levels of external noise, but increasingly so as external noise increases. In both $(a)$ and $(b)$, the magnitude of attention effects does not depend on the performance criterion. In (c), however, the magnitude of the attention effects depends critically on the performance criterion.

\subsubsection{External noise exclusion}

One-key way in which perceptual learning improves performance is by focusing perceptual analysis on the appropriate time, spatial region, and/or content characteristics of the signal stimulus. This focusing, or filtering, serves to eliminate external noise from further processing, and is related to claims by Shiu and Pashler (1994) and others. The behavioral signature for this mechanism is performance improvements in high external noise conditions (Fig. 2b). Mechanisms that filter external noise are of no benefit in the absence of the external noise. 


\subsubsection{Internal noise reduction}

Another possible mechanism of perceptual learning involves the reduction of internal noise. The reduction of additive noise is formally equivalent to the enhancement of stimulus (see above). Multiplicative noise increases with increasing contrast in the stimulus display. Reduction of multiplicative internal noise produces a signature of improvements in both high and low levels of external noise, with slightly larger effects in high external noise (Fig. 2c). To date, we have not empirically observed a case of multiplicative noise reduction by perceptual learning.

\subsubsection{Distinguish mechanism mixtures}

A direct comparison of the experimental data and the signature patterns of the PTM model may be sufficient to identify the underlying mechanisms of perceptual learning in certain situations. In other situations, mixtures of more than one mechanisms may underlie improvements in performance associated with perceptual learning in a task. In particular, a mixture of stimulus enhancement (low noise effects) and external noise exclusion (high noise effects) must be discriminated from multiplicative internal noise suppression (effects in low and high noise). This can be accomplished by measuring threshold versus external noise contrast functions at multiple criterion performance levels. A higher level of threshold performance, for example a $d$ ' of 1.5 instead of 1.0, requires higher contrast signals to achieve. For conditions differing in stimulus enhancement (Fig. 2a), or in external noise exclusion (Fig. 2b), the magnitude of the perceptual learning effect is the same (on the log contrast axis) at both the higher, more stringent and the lower, less stringent threshold performance levels. However, threshold contrast differences between the lower and higher criterion threshold values depend strongly upon criterion performance level in the conditions that differ in internal multiplicative noise reduction (Fig. 2c). Thus, measuring TvC functions at two or more criterion performance levels resolves the individual contribution of each mechanism in a mixture situation (Dosher and Lu, 1998, 1999).

\section{EMPIRICAL RESULTS}

\subsection{Initial applications}

In the first application of the external noise plus perceptual learning paradigm, Dosher and $\mathrm{Lu}$ $(1998,1999)$ investigated mechanisms of perceptual learning in an orientation identification task in the periphery. A concurrent letter identification task in fovea was used to demand eye fixation (Fig. 3a). Contrast thresholds in a range of external noise conditions were measured using two adaptive staircases (Fig. 3b, c) yielding $79.3 \%$ and $70.7 \%$ accuracies. We found that the threshold signal contrasts depend upon criterion accuracy, external noise level, and practice (Fig. 3b). Higher criterion accuracy demands higher contrast thresholds. In the high-noise region where external noise is the limiting factor in performance, contrast thresh- 
(a)

(b)
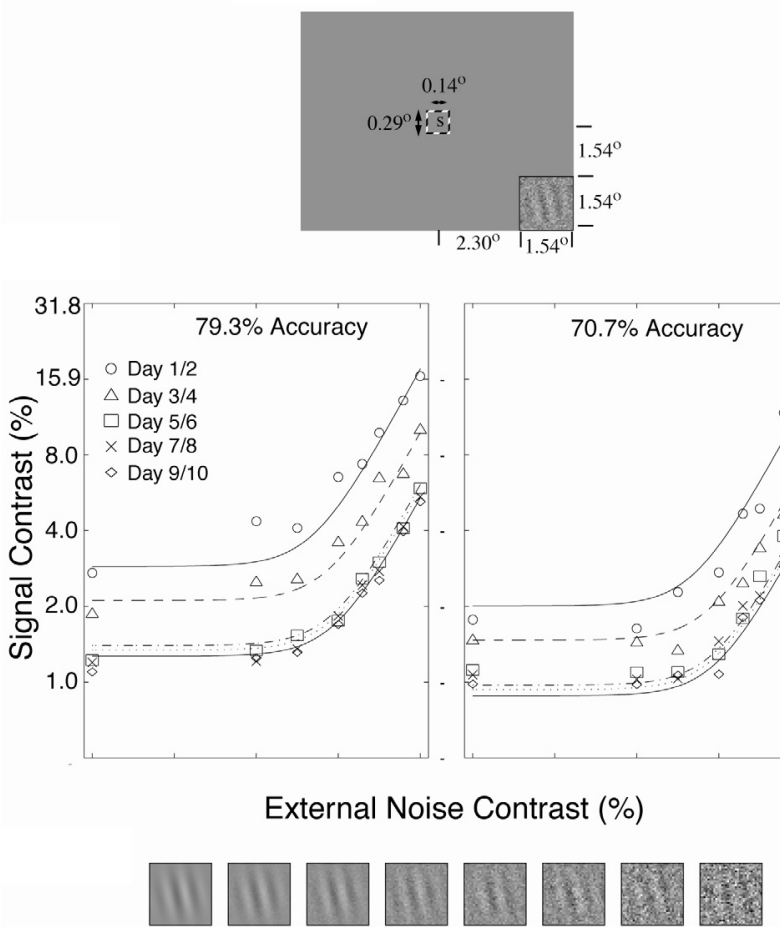

Figure 3. A perceptual learning task using the external noise paradigm. (a) Spatial layout of the task, including the peripheral orientation discrimination Gabor stimulus, and a central letter stimulus for a secondary task. (b) Contrast threshold (Gabor signal contrast corresponding to the criterion accuracy) as a function of the external noise in the stimulus. Threshold is a systematic function of criterion, external noise, and practice (data from Dosher and Lu, 1998). (c) Examples of a signal of constant contrast embedded in increasing amounts of external noise.

olds increase with increasing external noise. Practice reduces contrast thresholds by a downward vertical shift (in the log) with practice in both zero and in high external noise (albeit with slightly different magnitudes). We also observed a shift relationship between thresholds at the two performance criteria. These strong shift properties in the log contrast threshold as a function of criterion are a special characteristic of the perceptual template model. We concluded that perceptual learning improved performance (reduced contrast thresholds) at all levels of external noise and identified a mixture of stimulus enhancement and template retuning as the mechanisms of perceptual learning. Dosher and Lu (1999) performed detailed statistical analyses on their data and found that although performance improvements due to stimulus enhancement and template retuning co-occurred, the sizes of these separate improvements were only partially, not perfectly, coupled.

Using the same external noise technique but different signal stimuli, the same data pattern was replicated by Gold, Bennett and Sekuler (1999) for identification of band-pass noise samples and for novel face discrimination. Again, perceptual learning caused improvements in 
performance across both low and high external noise conditions. Although the data patterns were identical, Gold, Bennett and Sekuler (1999) concluded that, very differently from Dosher and $\mathrm{Lu}$ (1998), that perceptual learning enhances processing efficiency only for the signal stimulus. Gold, Bennett and Sekuler based their conclusions on the linear amplifier model, which has been shown to be too simple to account for human performance. Essentially, the linear amplifier model fails to give a consistent set of model estimates at different accuracy criteria - its estimates are very different at distinct points along a contrast psychometric function (see Lu and Dosher, 2008 for a review). Gold, Bennett and Sekuler (1999) also based their conclusion on an incorrect interpretation of the double-pass results.

The double-pass procedure was developed to directly estimate the total amount of internal noise, both additive and multiplicative, relative to external noise, in the perceptual system for each stimulus (signal and external noise) condition (Ahumada, 1967; Burgess and Colborne, 1988; Gilkey, Frank and Robinson, 1978, 1981; Green, 1964; Spiegel and Green, 1981). In the double-pass procedure, the same sequence of stimulus trials (signal + external noise) is repeated twice for each observer. Repeating each particular sample of external noise provides an assessment of the relative influence of the external and internal noises. Both response accuracy and response consistency (whether the response is or is not the same on the two identical tests) are measured across different passes of the same stimulus condition. If performance is only affected by external noise, this influence should be shared in the two repeated trials, leading to response consistency. The results of the double-pass experiments are traditionally (Burgess and Colborne, 1988) summarized in terms of probability correct $\left(P_{C}\right)$ versus probability agreement $\left(P_{A}\right)$ functions (Fig. 4). It is designed to estimate the ratio, $\alpha$, of the standard deviation of the total internal noise and that of the external noise. $\alpha$ completely determines the shape of the $P_{C}$ vs $P_{A}$ function in each external noise condition.

Gold, Bennett and Sekuler (1999) found that the $P_{C}$ vs $P_{A}$ functions were about the same before and after perceptual learning. They concluded that the internal noise did not change. However, this interpretation did not consider the fact that the standard deviation of the effective external noise depends on whether the perceptual template changes during perceptual

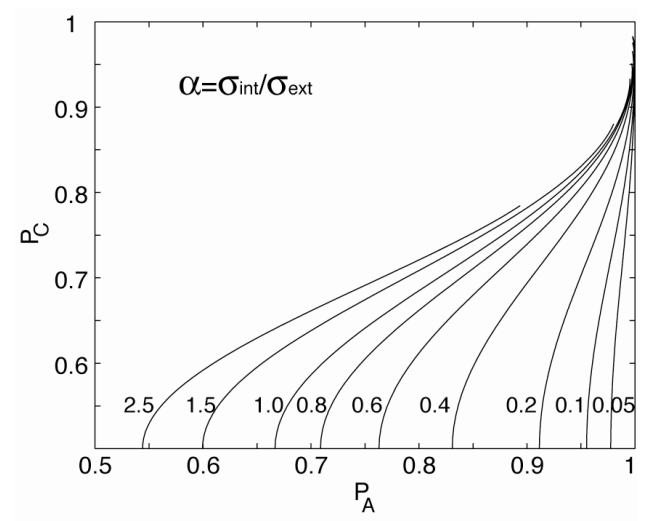

Figure 4. Probability correct $P_{c}$ vs probability consistent $P_{A}$ for a range of internal to external noise ratio $\alpha$ 's. 
learning. If perceptual learning retunes the perceptual template, then the effective external noise in the system is reduced. If the double-pass agreement function, and therefore the internal to external noise ratio did not change, then this implies that the internal noise was reduced approximately equivalently as the effective external noise. The conclusion that internal noise did not change is quite likely incorrect because it is based on the assumption that the template (or efficiency) was unchanged by perceptual learning - an assumption directly at odds with the primary conclusions of the study. In contrast, a mixture of stimulus enhancement and external noise exclusion, as observed in Dosher and $\mathrm{Lu}(1998,1999)$ fully accounts for the pattern of results in Gold, Bennett and Sekuler (1999).

\subsection{Pure mechanisms}

Pure mechanisms of perceptual learning, stimulus enhancement and external noise exclusion, have been documented in several studies.

$\mathrm{Lu}$ and Dosher (2004) trained observers in an orientation identification task (45 $\pm 8 \mathrm{deg})$ in fovea. TvC functions were measured across ten training sessions (Fig. 5). Significant learning

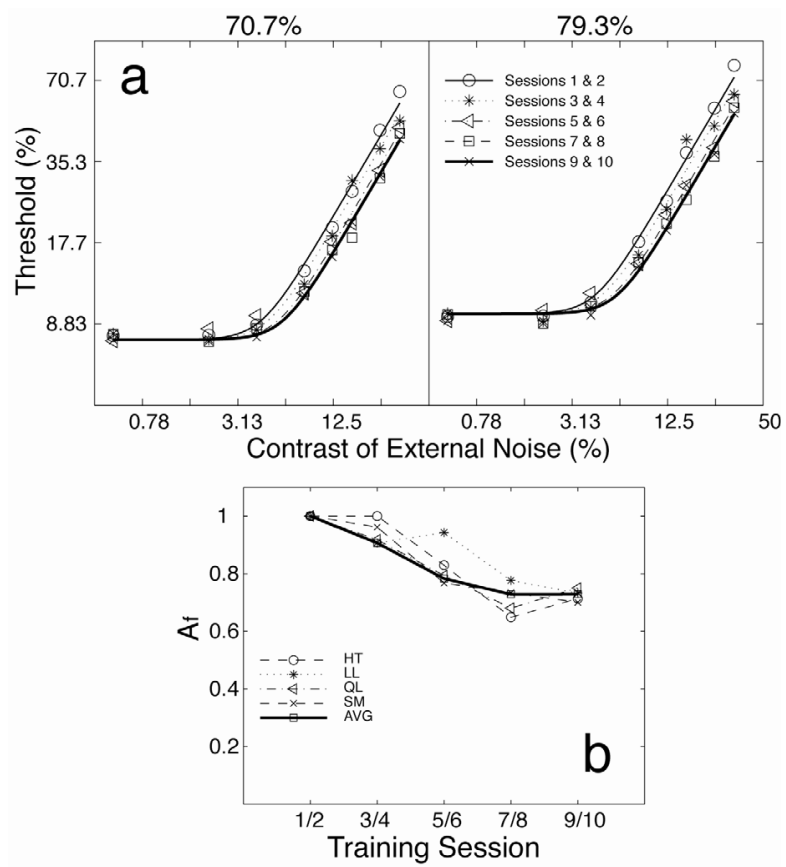

Figure 5. (a) Threshold versus external noise contrast (TvC) functions at two performance-criterion levels $(70.7 \%$ and $79.3 \%$ correct) over 10 training sessions, averaged across the four observers (Lu and Dosher, 2004). The smooth curves represent the best fit of the PTM model. The relative SEs of the thresholds are about 5\%. (b) versus training session blocks for the four observers as well as the "average" observer AVG. For the average observer AVG, reduced to 0.7289 after 10 sessions of practice. 
was only observed in the high external noise conditions but not in zero or low external noise conditions. We concluded that a pure mechanism of external noise exclusion provided the best account of the data. In fact, the observed pattern of perceptual learning - its dependence on the amount of external noise added to the signal stimulus - poses major challenges to the linear amplifier model-based accounts of perceptual learning (Gold, Bennett and Sekuler, 1999). Interpreted within this model, performance improvements in high external noise conditions require improved calculation efficiency in the LAM-based model, which would result in equivalent performance improvements (threshold reduction) across all the external noise levels. However, because no learning or less learning was observed in low external noise conditions, paradoxical compensatory increases of the equivalent internal noise that exactly counteract the improvements in calculation efficiency are necessary to account for the lack of perceptual learning in the low noise conditions. This plus the lack of a principled account of the calculation efficiency at different performance-criterion levels render the LAM-based theoretical framework both inconsistent and less parsimonious. In contrast, the PTM model provides a coherent account of the data in both attention and perceptual learning across multiple performance levels and task situations (Lu and Dosher, 2008; Tjan, Chung and Levi, 2002).

A pure mechanism of stimulus enhancement has been demonstrated in two experiments (Dosher and Lu, 2007; Kong et al., 2004). Dosher and Lu (2007) investigated the ability for observers to learn and improve their perceptual performance in $1^{\text {st }}$ order and in $2^{\text {nd }}$ order object (letter) orientation judgments. Surprisingly, (regular/mirror) orientation judgments for $1^{\text {st }}$ order letters at fovea were not consistently susceptible of improvement - whether in low or in high levels of external noise. Significant learning did occur for orientation judgments of letters defined by a ' 2 nd order' texture pattern, in which the letter was made from a checkerboard of light and dark squares. For these $2^{\text {nd }}$ order stimuli, the performance improvements predominantly occurred in low external noise conditions (Fig. 6). Similar patterns of results were also obtained in an auditory modulation detection experiment (Kong et al., 2004). In both cases, we concluded that perceptual learning affected the post-rectification stages of perceptual analysis. It serves to amplify the stimulus relative to limiting internal noise for intrinsically noisy representations of second-order stimuli.

\subsection{Independent mechanisms}

The separability of two mechanisms of perceptual learning, stimulus enhancement and external noise exclusion, was directly tested in two additional studies (Dosher and $\mathrm{Lu}, 2005$; $\mathrm{Lu}$, $\mathrm{Chu}$ and Dosher, 2006). Using an orientation identification task identical to Dosher and $\mathrm{Lu}$ (1998), Dosher and Lu (2005) found that training in a simple object orientation identification task exhibited an asymmetric pattern of transfer. Training with low noise exemplars transferred to high noise performance, while training with high noise exemplars - in which target objects were embedded in white external noise - did not transfer to low noise performance. They concluded that training in clear displays had unique advantages. They concluded that training improves the dominant limiting factor, which in the case of training in external noise involves improvements in filtering. 


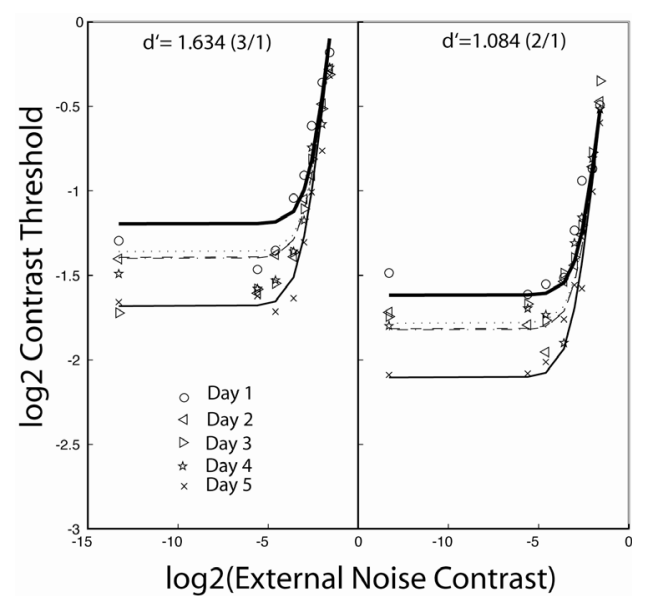

Figure 6. Contrast thresholds, averaged over observers, as a function of external noise contrast (TvC functions) for five practice days in the texture ( $2^{\text {nd }}$-order) letter task (Dosher and Lu, 2006). Data are shown for two criteria (3/1 and 2/1 staircases). The dark heavy lines are Day 1, and the lighter heavy lines are Day 5. Smooth curves are the fit of a PTM model with perceptual learning through stimulus enhancement.

$\mathrm{Lu}$, Chu and Dosher (2006) used pre-training as a manipulation to evaluate the separability of stimulus enhancement and external noise exclusion. Observers were trained in identifying the motion direction of a moving sine-wave grating in fovea with varying amount of superimposed external noise across trials, after receiving no pre-training, pre-training in high external noise, or pre-training in zero external noise in the same task. We found (1) Without pre-training, perceptual learning significantly reduced contrast thresholds by about the same amount across all the external noise levels. (2) Both types of pre-training significantly reduced contrast thresholds in the corresponding conditions. (3) Pre-training in high external noise greatly reduced subsequent learning in high external noise, accounting for $64.6 \%$ of the total (pre-training + subsequent) improvements in that condition. On the other hand, the amount of subsequent learning in low external noise conditions essentially the same as the total (pre-training + subsequent) amount of improvements in high external noise, suggesting that pre-training in high external noise had mostly only improved performance in noisy displays. (4) Pre-training in zero external noise practically eliminated or left very little additional learning in all the external noise conditions. We concluded that the two mechanisms of perceptual learning, stimulus enhancement and external noise exclusion, can be trained independently in motion direction discrimination in fovea; Training in low noise suffices to improve observer performance over all the external noise conditions.

That pre-training in high external noise is only effective in high external noise without impacting learning in low external noise completely rules out a single mechanism account of perceptual learning. In fact, any theoretical explanation of these results must invoke at least two independent mechanisms, one of which is only effective in high external noise. This dual mechanism account does not however require independent manifestation of the two inde- 


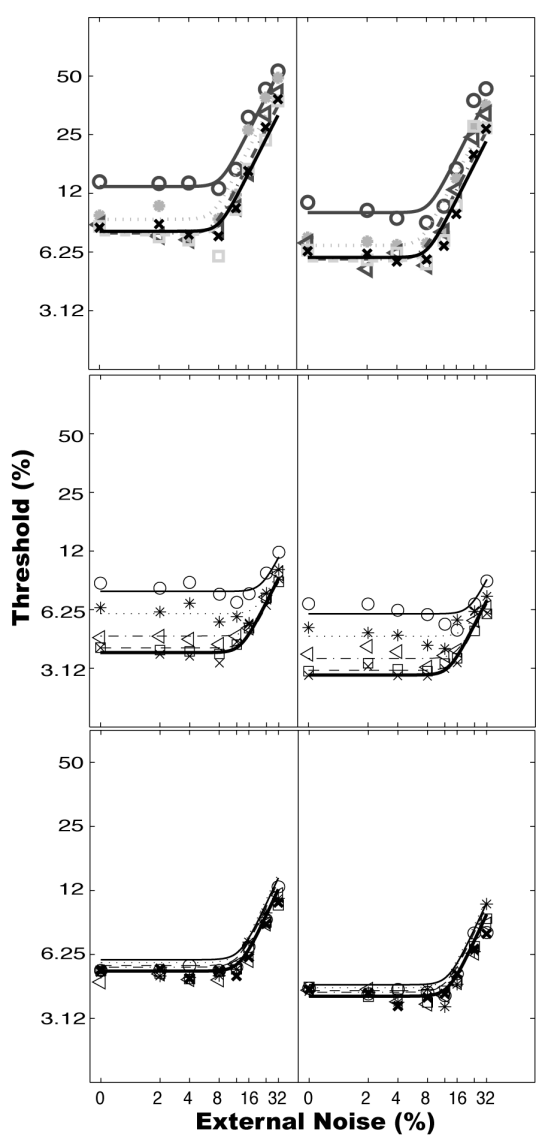

(a)

(b)

(c)

Figure 7. Average threshold versus external noise contrast (TvC) functions at two performance criterion levels (79.3\% and $70.7 \%$ correct) across ten training sessions in three experimental groups: (a) no pre-training, (b) pre-training in high external noise condition, and (c) pre-training in zero external noise condition.

pendent mechanisms in every circumstance - a particular training protocol could in fact train both mechanisms simultaneously and therefore improve both mechanisms together. Pretraining in high external noise only impacted the external noise external noise mechanism, but pre-training in zero noise impacted both stimulus enhancement and external noise exclusion. In the presence of high external noise, observer's performance is ONLY limited by external noise, not by internal noise. The only way to improve performance is to re-tune the perceptual template to eliminate external noise. That's how observers learned in pre-training and continued to benefit in subsequent learning. In zero external noise, observer's performance is limited by internal additive noise. They did indeed improve their performance via stimulus enhancement during pre-training and continue to benefit in low external noise during subsequent learning. In addition, the observers were also exposed to the signal stimuli and were ex- 
ercising the perceptual template. We suggest that the exposure to signal stimuli during pre-training in zero external noise condition allowed the observers to re-tune their perceptual templates (Seitz and Watanabe, 2003; Watanabe et al., 2002). Although the retuning did not directly benefit their performance during pre-training, it benefited performance in subsequent testing in high external noise.

\subsection{Transfer of mechanisms}

Dosher and Lu $(1998,1999)$ concluded that perceptual learning in an orientation identification task in peripheral vision is due to a mixture of stimulus enhancement and external noise exclusion. Dosher and Lu (1999) tested location specificity of both mechanisms by measuring $\mathrm{TvC}$ functions in new transfer locations. We found that improvements due to practice in the lower-right quadrant task did not transfer substantially to task performance in the transfer quadrants. Thresholds for the transfer tasks are higher than those for the practiced levels of the original task. Neither transfer condition differed significantly from the performance in the first practice set of the standard training; and both transfer conditions were reliably above the performance in the last practice set. Therefore the transfer test showed practice improvements in the orientation discrimination task, due to a mixture of both stimulus enhancement and external noise exclusion, were reasonably specific to the retinal location.

Lu et al. (2005) combined the external noise paradigm and transfer tests to investigate the mechanisms and eye specificity of perceptual learning of Gabor orientation in visual periphery. Using a task identical to Dosher and Lu (1998), TvC functions in one eye were measured over ten practice sessions, followed by five sessions of practice in the other eye to assess transfer. We found that monocular learning improved performance (reduced contrast thresholds) with virtually equal magnitude across a wide range of external noise levels with no significant change in central task performance. Moreover, perceptual learning in the trained eye generalized completely to the untrained eye. Based on physiology of binocular coding of orientation (Blasdel, 1992; Livingstone and Hubel, 1984), we suggest that the site for perceptual learning of Gabor orientation identification resides in areas post layer $4 \mathrm{Cb}$ of the primary visual cortex. This conclusion is largely consistent with observations of learning only post layer 4 in V1 by Schoups et al. (2001) and relatively small magnitude of learning in V1 neurons (Ghose, Yang and Maunsell, 2002) but relatively large amount of learning in largely binocular V4 neurons (Ghose, Yang and Maunsell, 2002; Yang and Maunsell, 2004) when monkeys performed orientation discrimination tasks.

Lu et al. (2005) characterized learning mechanisms in judging motion direction of moving luminance-defined objects in visual periphery (Experiment 1) and fovea (Experiment 2) and investigated the degree of transfer of the mechanisms of learning from the trained to the untrained eyes. We found that learning in the trained eye improved performance in that eye with virtually equal magnitude across a wide range of external noise levels. Interestingly, the degree of transfer depended on the amount of external noise added to the signal stimuli. In high external noise conditions, learning transferred completely to the untrained eye. In low external noise conditions, there was only partial transfer of learning: 63\% in Experiment 1 and 54\% in Experiment 2. Subsequent practice in the untrained eye further improved performance via 
stimulus enhancement in transfer sessions. We concluded that independent mechanisms underlie perceptual learning of motion direction identification in monocular and binocular motion systems.

According to Lu and Sperling (2001), there are two monocular and one (less sensitive) binocular luminance motion systems; All three systems provide inputs to motion integration and decision on motion direction. One possible interpretation of the relative sensitivities of the monocular and binocular motion systems is that the monocular systems have less internal noise and the binocular system is limited by higher amount of internal noise. Training in one eye affects both the corresponding monocular motion system and the binocular motion system. In displays with no or little added external noise, both the monocular and the binocular motion systems are limited by internal noise; effective learning should mostly result from further reduction of the internal noise in the more sensitive monocular motion system. Because the relative sensitivity of the monocular and binocular system is about 2 to 1 (Lu and Sperling, 2001), not infinity, a certain amount of training of the binocular system is of course inevitable. This could account for the observed partial transfer of learning from the trained to the untrained eyes. In high external noise displays, the monocular and binocular motion systems are mostly limited by external rather than internal noise; learning via template retuning improves both systems. Our results suggest that the binocular system might be more susceptible to template retuning, consistent with recent neurophysiological results in largely binocular MT neurons (Bisley and Pasternak, 2000).

\section{DISCUSSION AND CONCLUSIONS}

Using the external noise paradigm and the perceptual template model, we have documented two mechanisms of perceptual learning, stimulus enhancement and external noise exclusion. It appears that these two mechanisms either together or alone explain most perceptual learning studied so far. In different experiments that used different training tasks, we documented both coupled and pure mechanisms. Moreover, we showed that the two mechanisms are independent in studies that manipulated pre-training conditions. Both mechanisms are shown to be specific to retinal location but not specific to the eye of training in an orientation identification task. However, stimulus enhancement is partially specific to the training eye in motion direction discrimination.

We suggest that perceptual learning primarily serves to select or strengthen the appropriate channel and prune or reduce inputs from irrelevant channels. The connections between the most closely tuned visual channel and a learned categorization structure are maintained or strengthened, while input from other channels is reduced or eliminated. A similar interpretation has been used to account for the improvements in a single stimulus relative to mixed stimulus blocks in uncertainty experiments (Graham, 1989). Reducing the weights on irrelevant channels reduces the contributions of external noise and additive internal noise. Perceptual learning, then, reflects plasticity in the relative activity of different basic visual channels, which contribute to categorization. At some level of neural representation, this might appear as a reorganization in the weighting of the critical channel in cortical maps (e.g. Recanzone, Schreiner and Merzenich, 1993).

Learning \& Perception 1 (2009) 1 
In summary, we find evidence that two independent mechanisms, external noise exclusion and stimulus enhancement support perceptual learning across a range of tasks. We suggest that both mechanisms may reflect re-weighting of stable early sensory representations.

\section{ACKNOWLEDGEMENTS}

This research was supported by the National Science Foundation, National Institute of Mental Health, and National Eye Institute.

\section{REFERENCES}

Ahissar, M., Hochstein, S. (1996): Learning pop-out detection: Specificities to stimulus characteristics. Vision Research, 36(21), 3487-3500.

Ahissar, M., Hochstein, S. (1997): Task difficulty and the specificity of perceptual learning. Nature, 387(6631), 401-406.

Ahissar, M., Laiwand, R., Kozminsky, G., Hochstein, S. (1998): Learning pop-out detection: Building representations for conflicting target-distractor relationships. Vision Research, 38(20), 3095-3107.

Ahumada, A. J. (1967): Detection of Tones Masked by Noise: A Comparison of Human Observers with Digital-Computer-simulated Energy Detectors of Varying Bandwidths. Los Angeles: University of California.

Ball, K., Sekuler, R. (1982): A specific and enduring improvement in visual motion discrimination. Science, 218(4573), 697-698.

Ball, K., Sekuler, R. (1987): Direction-specific improvement in motion discrimination. Vision Research, 27(6), 953-965.

Beard, B. L., Levi, D. M., Reich, L. N. (1995): Perceptual learning in parafoveal vision. Vision Research, 35(12), 1679-1690.

Berardi, N., Fiorentini, A. (1987): Interhemispheric transfer of visual information in humans: Spatial characteristics. Journal of Physiology, 384, 633-647.

Bisley, J. W., Pasternak, T. (2000): The multiple roles of visual cortical areas MT/MST in remembering the direction of visual motion. Cerebral Cortex, 10(11), 1053-1065.

Blasdel, G. G. (1992): Orientation selectivity, preference, and continuity in monkey striate cortex. Journal of Neuroscience, 12(8), 3139-3161.

Burgess, A. E., Colborne, B. (1988): Visual signal detection: IV. Observer inconsistency. Journal of the Optical Society of America a-Optics Image Science and Vision, 2, 617-627.

Chung, S. T. L., Levi, D. M., Tjan, B. (2005): Learning letter identification in peripheral vision. Vision Research, 45(11), 1399-1412.

Crist, R. E., Li, W., Gilbert, C. D. (2001): Learning to see: Experience and attention in primary visual cortex. Nature Neuroscience, 4(5), 519-525.

DeValois, K. (1977): Spatial frequency adaptation can enhance contrast sensitivity. Vision Research, 17, 1057-1065.

Dorais, A., Sagi, D. (1997): Contrast masking effects change with practice. Vision Research, 37(13), 1725-1733.

Dosher, B., Lu, Z.-L. (2007): Level and mechanisms of perceptual learning: Learning in luminance and texture objects. Vision Research, 46, 2007. 
Dosher, B. A., Lu, Z.-L. (1998): Perceptual learning reflects external noise filtering and internal noise reduction through channel reweighting. Proceedings of the National Academy of Sciences USA, 95(23), 13988-13993.

Dosher, B. A., Lu, Z.-L. (1999): Mechanisms of perceptual learning. Vision Research, 39(19), 31973221.

Dosher, B. A., Lu, Z.-L. (2005): Perceptual learning in clear displays optimizes perceptual expertise: Learning the limiting process. Proceedings of the National Academy of Sciences USA, 102(14), $5286-5290$.

Fahle, M., Daum, I. (2002): Perceptual learning in amnesia. Neuropsychologia, 40(8), 1167-1172.

Fahle, M., Edelman, S. (1993): Long-term learning in vernier acuity: Effects of stimulus orientation, range and of feedback. Vision Research, 33(3), 397-412.

Fine, I., Jacobs, R. A. (2000): Perceptual learning for a pattern discrimination task. Vision Research, 40(23), 3209-3230.

Fiorentini, A., Berardi, N. (1980): Perceptual learning specific for orientation and spatial frequency. $\mathrm{Na}$ ture, 287(5777), 43-44.

Fiorentini, A., Berardi, N. (1981): Learning in grating waveform discrimination: Specificity for orientation and spatial frequency. Vision Research, 21(7), 1149-1158.

Furmanski, C. S., Engel, S. A. (2000): Perceptual learning in object recognition: Object specificity and size invariance. Vision Research, 40(5), 473-484.

Ghose, G. M., Yang, T., Maunsell, J. H. R. (2002): Physiological correlates of perceptual learning in monkey V1 and V2. Journal of Neurophysiology, 87(10), 1867--1888.

Gilkey, R. H., Frank, A. S., Robinson, D. E. (1978): Estimates of internal noise. Journal of the Acoustical Society of America, 64, S36 (A).

Gilkey, R. H., Frank, A. S., Robinson, D. E. ( 1981): Estimates of the ratio of external to internal noise obtained using repeatable samples of noise. Journal of the Acoustical Society of America, 69, S23 (A).

Gold, J., Bennett, P. J., Sekuler, A. B. (1999): Signal but not noise changes with perceptual learning. Nature, 402(6758), 176-178.

Graham, N. V. S. (1989): Visual Pattern Analyzers. New York, NY, US: Oxford University Press, pp. xvi, 646.

Green, D. M. (1964): Consistency of auditory detection judgments. Psychological Review, 71, 392-407.

Karni, A., Sagi, D. (1991): Where practice makes perfect in texture-discrimination - Evidence for primary visual-cortex plasticity. Proceedings of the National Academy of Sciences USA, 88(11), 4966-4970.

Karni, A., Sagi, D. (1993): The time course of learning a visual skill. Nature, 365(6443), 250-252.

Kong, Y.-Y., Lu, Z.-L., Dosher, B., Zeng, F.-G. (2004): Mechanisms of Perceptual Learning in Amplitude Modulation Detection. Association for Research in Otolaryngology Mid-Winter Meeting (Florida).

Liu, Z., Vaina, L. M. (1998): Simultaneous learning of motion discrimination in two directions. Cognitive Brain Research, 6(4), 347-349.

Livingstone, M. S., Hubel, D. H. (1984): Specificity of intrinsic connections in primate primary visual cortex. Journal of Neuroscience, 4(11), 2830-2835.

Lu, Z.-L., Chu, W., Dosher, B., Lee, S. (2005): Perceptual learning of Gabor orientation identification in visual periphery: Complete inter-ocular transfer of learning mechanisms. Vision Research, 45, 2500-2510.

Lu, Z.-L., Chu, W., Dosher, B. A. (2006): Perceptual learning of motion direction discrimination in fovea: Separable mechanisms. Vision Research, 46, 2315-2327.

Lu, Z.-L., Chu, W., Dosher, B. A., Lee, S. (2005): Independent perceptual learning in monocular and binocular motion systems. Proceedings of the National Academy of Sciences USA, 102, 5624-5629. 
Lu, Z.-L., Dosher, B. (2008): Characterizing observer states using external noise and observer models: Assessing internal representations with external noise. Psychological Review, 115(1), 44-82.

Lu, Z.-L., Dosher, B. A. (1999): Characterizing human perceptual inefficiencies with equivalent internal noise. Journal of the Optical Society of America a-Optics Image Science and Vision, 16(3), 764-778.

Lu, Z.-L., Dosher, B. A. (2004): Perceptual learning retunes the perceptual template in foveal orientation identification. Journal of Vision, 4, 44-56.

Lu, Z.-L., Sperling, G. (2001): Three-systems theory of human visual motion perception: review and update. Journal of the Optical Society of America a-Optics Image Science and Vision, 18(9), 2331-2370.

Lu, Z.-L., Xu, P., Wang, X., Dosher, B. A., Zhou, J., Zhang, D., Zhou, Y. (2008): Category and perceptual learning in subjects with treated Wilson's disease. http://journalofvision. org/8/6/977/

Mayer, M. (1983): Practice improves adults' sensitivity to diagonals. Vision Research, 23, 547-550.

McKee, S. P., Westheimer, G. (1978): Improvement in vernier acuity with practice. Perception \& Psychophysics, 24(3), 258-262.

Mollon, J. D., Danilova, M. V. (1996): Three remarks on perceptual learning. Spatial Vision, 10(1), $51-58$.

Moran, J., Desimone, R. (1985): Selective attention gates visual processing in the extra-striate cortex. Science, 229, 782-784.

Pelli, D. (1981): Effects of visual noise. PhD. disseration. University of Cambridge, Cambridge, England.

Poggio, T., Fahle, M., Edelman, S. (1992): Fast perceptual learning in visual hyperacuity. Science, 256(5059), 1018-1021.

Ramachandran, V. S., Braddick, O. (1973): Orientation-specific learning in stereopsis. Perception, 2(3), 371-376.

Recanzone, G. H., Schreiner, C. E., Merzenich, M. M. (1993): Plasticity in the frequency representation of primary auditory cortex following discrimination training in adult owl monkeys. Journal of Neuroscience, 13(1), 87-103.

Reynolds, J. H., Pasternak, T., Desimone, R. (2000): Attention increases sensivity of V4 neurons. Neuron, 26(6), 703-714.

Rubenstein, B. S., Sagi, D. (1993): Effects of foreground scale in texture discrimination tasks: Performance in size, shape, and content specific. Spatial Vision, 7(4), 293-310.

Saarinen, J., Levi, D. M. (1995): Perceptual learning in vernier acuity: What is learned? Vision Research, 35(4), 519-527.

Sagi, D., Tanne, D. (1994): Perceptual-learning - learning to see. Current Opinion in Neurobiology, 4(2), 195-199.

Schiltz, C., Bodart, J. M., Dubois, S., Dejardin, S., Michel, C., Roucoux, A., Crommelinck, M., Orban, G. (1999): Neuronal mechanisms of perceptual learning: Changes in human brain activity with training in orientation discrimination. NeuroImage, 9, 46-62.

Schoups, A., Vogels, R., Qian, N., Orban, G. (2001): Practising orientation identification improves orientation coding in V1 neurons. Nature, 412(6846), 549-553.

Schoups, A. A., Vogels, R., Orban, G. A. (1995): Human perceptual learning in identifying the oblique orientation: Retinotopy, orentation specificity and monocularity. Journal of Physiology, 483, 797-810.

Schwartz, S., Maquet, P., Frith, C. (2002): Neural correlates of perceptual learning: A functional MRI study of visual texture discrimination. Proceedings of the National Academy of Sciences USA, 99, 17137-17142.

Seitz, A. R., Watanabe, T. (2003): Is subliminal learning really passive? Nature, 422(6927), 36-36. 
Shiu, L.-P., Pashler, H. (1992): Improvement in line orientation discrimination is retinally local but dependent on cognitive set. Perception \& Psychophysics, 52(5), 582-588.

Shiu, L.-P., Pashler, H. U. S. (1994): Negligible effect of spatial precuing on identification of single digits. Journal of Experimental Psychology: Human Perception \& Performance, 20(5), 1037-1054.

Sperling, G. (1989): Three stages and two systems of visual processing. Spatial Vision, 4(2-3), 183-207.

Spiegel, M. F., Green, D. M. (1981): Two procedures for estimating internal noise. Journal of the Acoustical Society of America, 70, 69-73.

Tjan, B. S., Chung, S., Levi, D. (2002): Limitation of ideal-observer analysis in understanding perceptual learning. Twenty-seventh Annual Interdisciplinary Conference (Jackson Hole, Wyoming).

Treue, S., Maunsell, J. H. R. (1999): Effects of attention on the processing of motion in macaque middle temporal and medial superior temporal visual cortical areas. Journal of Neuroscience, 19(17), 7591-7602.

Vogels, R., Orban, G. A. (1985): The effect of practice on the oblique effect in line orientation judgments. Vision Research, 25(11), 1679-1687.

Watanabe, T., Nanez, J. E. S., Koyama, S., Mukai, I., Liederman, J., Sasaki, Y. (2002): Greater plasticity in lower-level than higher-level visual motion processing in a passive perceptual learning task. Nature Neuroscience, 5(10), 1003-1009.

Yang, T., Maunsell, J. H. R. (2004): The effect of perceptual learning on neuronal responses in monkey visual area V4. Journal of Neuroscience, 24(7), 1617-1626. 